\title{
Minimal dose requirements for nasal mupirocin and its role in the control of epidemic MRSA
}

\author{
M. W. Casewell and R. L. R. Hill
}

\author{
Department of Medical Microbiology, King's College School of Medicine $\xi^{\circ}$ \\ Dentistry, Denmark Hill, London SE5 8RS, UK
}

\begin{abstract}
Summary: Staphylococci are still a leading cause of hospital infection. The success of nasal mupirocin for the control of epidemic methicillin-resistant Staphylococcus aureus (EMRSA), the prevention of colonization of central venous cannulae, and the prevention of septicaemia in haemodialysis patients should encourage the use of minimal dose regimens to minimize the emergence of mupirocin resistance. Mupirocin applied to the anterior nares 4times daily usually eliminates $S$. aureus, including EMRSA, within $48 \mathrm{~h}$. Elimination is sustained for several weeks in patients and staff. We recently found that a single dose, or a regimen of 4-times daily for 2 days, eliminated nasal carriage of $S$. aureus within $24 \mathrm{~h} ; 7$ days after a single dose, $92 \%$ of the subjects were still cleared; 7 days after the 2-day course, $96 \%$ remained free of nasal $S$. aureus. Ward personnel who are nasal carriers of EMRSA can, provided that other carriage sites are negative, return to work after 2 days of a 4-times daily intranasal regimen. The UK guidelines, recently published in this Journal, recommend an aggressive approach to identifying and eliminating EMRSA, including the elimination of nasal carriage. This approach is increasingly associated with the control of EMRSA in the UK and elsewhere.
\end{abstract}

Keywords: Mupirocin; nasal application; Staphylococcus aureus; colonization; eradication.

\section{Introduction}

Staphylococci continue to be a leading cause of hospital-acquired infection. There is increasing worldwide concern about the morbidity, mortality and cost of hospital outbreaks of strains of methicillin-resistant Staphylococcus aureus that have enhanced epidemicity ('EMRSA'), and about coagulase-negative staphylococcal infection of implanted prosthetic devices. The anti-staphylococcal activity of mupirocin provides new opportunities to control and to prevent such infections, including the containment of EMRSA, ${ }^{1-4}$ the early colonization of central venous cannulae $^{5}$ and the prevention of haemodialysis septicaemia $a^{6}$ and recurrent furunculosis. As with all antimicrobial agents, inappropriate or unnecessarily prolonged therapy with mupirocin may sooner, rather than later, result in the emergence of resistance and loss of efficacy. In view of the initial successes of mupirocin, it seems appropriate to review the evidence suggesting that relatively short-term therapy with the intranasal preparation ('Bactroban Nasal') may be effective, particularly in the context of the control of EMRSA. 


\section{Importance of nasal carriage as a source of infection with $S$. aureus}

Although nasal carriage of $S$. aureus is usually harmless, it was shown more than 30 years ago that nasal carriers are more prone to surgical wound infection and in approximately half of these the infecting strain is derived from the patient's own nose. ${ }^{7,8}$ Other examples of auto-infection from nasal $S$. aureus include bacteraemia in haemodialysis patients ${ }^{6}$ and recurrent furunculosis. Nasal carriage of EMRSA can provide a source of EMRSA for the patient's fingers ( $R$. Wenzel, personal communication) and probably other skin sites and wounds. ${ }^{9}$

In addition to auto-infection, it was also shown in the 1960 s that nasal carriage by patients and staff of strains of $S$. aureus with enhanced epidemicity (analogous to EMRSA) provided a significant source for nasal acquisition by other, previously non-colonized, patients. ${ }^{10,11}$ The early availability and use of intranasal mupirocin in the UK has been associated with the, hitherto unusual, successful control of EMRSA outbreaks by ourselves and by others. ${ }^{1-4}$

\section{Evidence for efficacy of short-term nasal mupirocin for elimination of nasal $S$. aureus}

From the outset in 1985, our published anecdotal observations on the first five patients and five nurses treated with a 5-day course of intranasal mupirocin for the elimination of nasal EMRSA (all of whom had failed with 10 days' nasal application of $1 \%$ obstetric chlorhexidine) indicated that mupirocin was uniformly effective. ${ }^{12}$ Chlorhexidine fails, despite the minimum inhibitory concentration (MIC) of $2.0 \mathrm{mg} \mathrm{l}^{-1}$, probably because it is negatively charged and is inactivated by nasal secretions which carry the opposite polarity. Using a bioassay method we found no such inactivation of mupirocin by nasal secretions (Hill \& Casewell, unpublished observations).

Two parameters are important when assessing the efficacy of any regimen for clearing nasal carriage of $S$. aureus: the number of days therapy to achieve clearance and the post-treatment interval to recolonization. In a blind controlled trial, $2 \%$ mupirocin in soft white paraffin and lanolin given 4-times a day to 32 healthy volunteers eliminated nasal $S$. aureus in all subjects within 2 days of a 5 -day course. ${ }^{13}$ For all subjects, nasal $S$. aureus was eliminated for at least 2 weeks after the course, and even after 5 weeks only six $(20 \%)$ had resumed carriage. Of those who ultimately resumed carriage, phage typing and antibiograms identified only $29 \%$ of subjects as having a relapse of colonization with the pre-treatment strain. None of these isolates showed an increase in the MIC of mupirocin. Soon after treatment the nasal flora was predominantly coagulase-negative staphylococci, coryneforms, or both. Minority populations of micrococci, Bacillus spp., Pseudomonas, Proteus or Escherichia coli also occurred but there was no overgrowth with mupirocin-resistant $S$. aureus or with yeasts or Gram-negative bacilli. The MICs of mupirocin for post-treatment 
coagulase-negative staphylococci were always $<0.25 \mathrm{mg}^{-1}$, an important observation as these organisms are a likely source of genetic elements that code for high-level mupirocin resistance in $S$. aureus. ${ }^{14}$

A large hospital-wide outbreak of EMRSA in London in the early 1980s provided enough patients and staff with stable nasal colonization with EMRSA for us to confirm the efficacy of mupirocin against nasal EMRSA. Of 40 patients and 32 hospital staff, all but one patient and one staff member were cleared of nasal EMRSA within the first $48 \mathrm{~h}$ of a 5 -day course. ${ }^{9}$ Recolonization with EMRSA was uncommon and delayed: of the 40 patients, 36 remained clear until discharge 1-9 weeks later and four became recolonized 1-5 weeks after the course. Of the 32 staff, all were negative one week after the course and of the 22 still available for follow up 8 weeks after the course, all were consistently negative.

These results for S. aureus, including EMRSA, suggested that 2, and certainly 3 , days of mupirocin applied to the anterior nares four times a day is adequate to clear nasal carriage in all subjects. The exposure of $S$. aureus to mupirocin could be reduced still further by fewer applications per day or by reducing the number of days of treatment. We therefore conducted a blind controlled trial in which we compared the effect of a single dose of mupirocin with a regimen of 4-times daily for 2 days among 44 volunteers (mainly medical and nursing students) who had stable nasal carriage of methicillin-sensitive $S$. aureus. Figure 1 summarizes these results. The single dose was as effective as 4 doses at eliminating carriage in all subjects within the first $24 \mathrm{~h}$. Seven days after the application of one dose and eight doses, $92 \%$ and $96 \%$ of the subjects, respectively, were still clear of nasal S. aureus.

If these findings were true for EMRSA, hospital personnel with nasal EMRSA may be able to return to work $24 \mathrm{~h}$ after a single dose, but to sustain this clearance, 8 doses over $48 \mathrm{~h}$ will be required which, 7 days after the course, should ensure that $96 \%$ of them would still be negative.

These findings will doubtless be relevant to those attempting elimination of nasal carriage in haemodialysis patients by giving mupirocin as an intermittent regimen. ${ }^{6}$

\section{The role of mupirocin in the control of EMRSA}

A revised version of the previous guidelines for the control of epidemic MRSA, drawn up by the Combined Working Party of the Hospital Infection Society and the British Society for Antimicrobial Chemotherapy, has been published recently in this Journal. ${ }^{15}$ These guidelines recommend the use of mupirocin for the successful control of EMRSA. The essential features of these guidelines are summarized as follows:

1. Following the recognition of two or more patients with MRSA in a general ward, or one patient in a ward of high-risk patients (ICU etc.), there should be thorough and complete identification (by at least nasal screening) of all cases and carriers, including staff nasal carriers. 


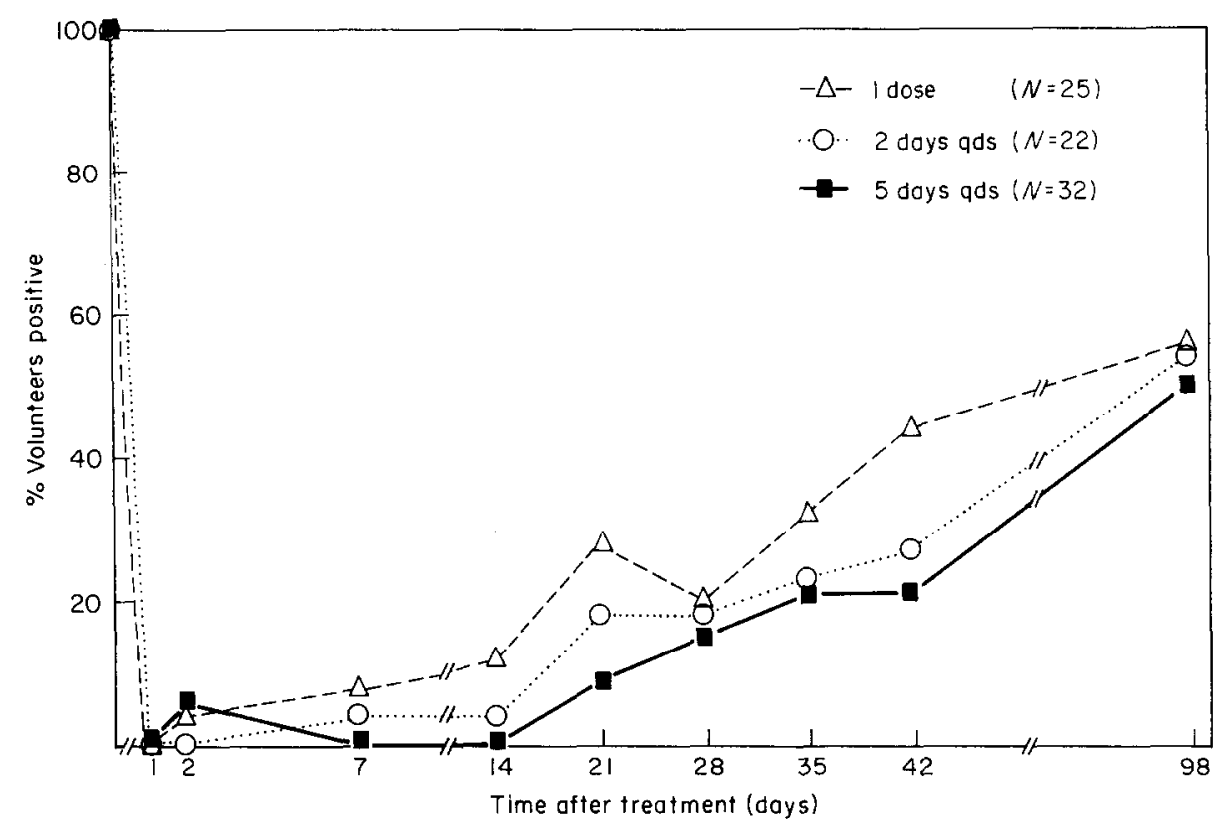

Figure 1. Effect of mupirocin on nasal carriage of Staphylococcus aureus.

2. By typing with experimental phages, it is possible to determine whether the strain of MRSA is known to have enhanced epidemic potential, i.e. is 'EMRSA'. In the UK this valuable information is provided by the Staphylococcal Reference Laboratory of the Public Health Laboratory Service at Colindale.

3. An aggressive programme to eliminate all patient and staff carrier sites, to include the use of mupirocin to eliminate nasal carriage, should be initiated promptly.

Our findings on the efficiency of short courses described below should help hospital infection control personnel rationalize the minimal dose regimens of mupirocin for clearance of nasal carriage and indicate the timing of subsequent follow-up nasal swabs that are required to check the continued clearance of treated carriers.

Newly identified staff nasal carriers should have other sites (skin lesions, throat, axillae and perineum) screened for MRSA and they should then start nasal mupirocin 4-times daily for 5 days and be taken off duty. Provided that these other sites are negative, they can return to work after only $48 \mathrm{~h}$ with the certainty of cleared nasal carriage. This regimen will ensure nasal clearance for at least 7-14 days after the course ${ }^{10}$ and re-screening for recolonization of the nose with MRSA need only be carried out weekly. This regimen carries significant cost savings in terms of lost working days.

At King's College Hospital, with the early availability of mupirocin, we have, since 1985 , adopted these principles ${ }^{16}$ with some success. Table I 
Table I. Epidemic $M R S A$ ('EMRSA-1') in a liver transplant unit

\begin{tabular}{lcc}
\hline Year & $\begin{array}{c}\text { No. } \\
\text { index } \\
\text { cases }\end{array}$ & $\begin{array}{c}\text { No. } \\
\text { secondary } \\
\text { cases* }\end{array}$ \\
\hline 1985 & 1 & 1 \\
1986 & 1 & 4 \\
1987 & 2 & 0 \\
1988 & 0 & 0 \\
1989 & 2 & 4 \\
Total & 6 & 9 \\
\hline
\end{tabular}

* All colonization, no infections.

shows, as an example, the repeated introduction of a strain of MRSA of known epidemic and pathogenic potential ('EMRSA-1') into a ward of highly immunocompromised liver-transplant patients. There have, to date, been only nine secondary transmissions and all have been recognized and contained before colonization became clinical infection. Others are also now reporting similar success by using mupirocin in this way. ${ }^{2-4}$ Early recognition and definition of outbreaks and an aggressive approach to elimination of the organism is, even in countries where mupirocin is unavailable and not used, also meeting with some success, albeit with more difficulty. ${ }^{17}$

\section{References}

1. Casewell MW. The successful control of MRSA using screening, typing and mupirocin. Abstract No. A62. Third International Conference on Nosocomial Infection. Atlanta, Georgia, August 1990.

2. Dacre JE, Emmerson AM, Jenner EA. Gentamicin-methicillin-resistant Staphylococcus aureus: epidemiology and containment of an outbreak. F Hosp Infect 1986; 7: 130-136.

3. Davies EA, Emmerson AM, Hogg EM, Patterson MF, Shields MD. An outbreak of infection with a methicillin-resistant Staphylococcus aureus in a special care baby unit: value of topical mupirocin and of traditional methods of infection control. $y$ IIosp Infect 1987; 10: 120-128.

4. Barrett SP. The value of mupirocin in containing an outbreak of methicillin-resistant Staphylococcus aureus in an orthopaedic unit. F Hosp Infect 1990; 15: 137-142.

5. Hill RLR, Fisher AP, Ware AJ, Wilson S, Casewell MW. Mupirocin for the reduction of colonization of internal jugular cannulae-a randomized controlled trial. $\mathcal{f}$ Hosp Infect 1990; 15: 311-21.

6. Boelaert JR, De Barre Y, Geernaert M, Godard C, Van Landuyt HW. Nasal mupirocin to prevent Staphylococcus aureus bacteraemias in haemodialysis patients. Cost-benefit analysis. Abstract No. SS4/6. Second International Conference of the Hospital Infection Society. London, September 1990.

7. Williams REO. Healthy carriage of Staphylococcus aureus: its prevalence and importance. Bact Rev 1963; 27: 56-71.

8. Casewell MW, Hill RLR. The carrier state: methicillin-resistant Staphylococcus aureus. F Antimicrob Chemother 1986; 18 (Suppl. A): 1-12.

9. Hill RLR, Duckworth GJ, Casewell MW. Elimination of nasal carriage of methicillin-resistant Staphylococcus aureus with mupirocin during a hospital outbreak. $\mathcal{f}$ Antimicrob Chemother 1988; 22: 377-384. 
10. Lidwell OM, Polakoff S, Jevons MP et al. Staphylococcal infection in thoracic surgery: experience in a subdivided ward. F Hyg (Camb) 1966; 64: 321-337.

11. Lidwell OM, Polakoff S, Davies J et al. Nasal acquisition of Staphylococcus aureus in a subdivided and mechanically ventilated ward: endemic prevalence of a single staphylococcal strain. F Hyg (Camb) 1970; 68: 417-433.

12. Casewell MW, Hill RLR. The effect of mupirocin (pseudomonic acid) on the nasal carriage of Staphylococcus aureus. In: Dobson RL, Noble WC, Price JD, Eds. Bactroban (Mupirocin). Excerpta Medica Current Clinical Practice Series 16. 1985, 47-53.

13. Casewell MW, Hill RLR. Elimination of nasal carriage of Staphylococcus aureus with mupirocin ('pseudomonic acid') - a controlled trial. 7 Antimicrob Chemother 1986; 17: $365-372$.

14. Hill RLR, Kader A, Casewell MW. The origin of high-level mupirocin resistance amongst staphylococci. Abstract P1/10. Second International Conference of the Hospital Infection, Society. London, September 1990.

15. Report. Revised guidelines for the control of epidemic methicillin-resistant Staphylococcus aureus. Report of a combined working party of the Hospital Infection Society and British Society for Antimicrobial Chemotherapy. $\mathcal{F}$ Hosp Infect 1990; 16: $351-377$

16. Casewell MW. Epidemiology and control of the 'modern' methicillin-resistant Staphylococcus aureus. $\mathcal{F}$ Hosp Infect 1986; 7 (Suppl. A): 1-11.

17. Vandenbroucke-Grauls CMJE, Frenay HME, Verhoef J. Spread and control of methicillin-resistant Staphylococcus aureus in a Dutch University Hospital. Abstract F1/9. Second International Conference of the Hospital Infection Society. London, September 1990. 